\title{
Endoprothetischer Gelenkersatz und Sport
}

\section{Total Joint Replacement and Return to Sports}

Autoren

Institut
N. Oehler, T. Schmidt, A. Niemeier

Klinik und Poliklinik für Orthopädie, Universitätsklinikum Hamburg-Eppendorf, Hamburg
Schlüsselwörter

- Endoprothese

- Implantation

- Sport

\section{Key words}

- endoprosthesis

implantation

- sport

\section{Bibliografie}

DOI http://dx.doi.org/ 10.1055/s-0042-119109 Sportverl Sportschad 2016; 30: 195-203 @ Georg Thieme Verlag KG Stuttgart · New York ISSN 0932-0555

\section{Korrespondenzadresse}

Prof. Andreas Niemeier Universitätsklinikum HamburgEppendorf

Martinistraße 52

20246 Hamburg

Deutschland

niemeier@uke.de

\section{Zusammenfassung \\ $\nabla$}

Hintergrund: Es finden sich zunehmend Patienten mit sportlichem Funktionsanspruch, für die nicht nur die Frage nach grundsätzlicher Sportfähigkeit nach Endoprothesenimplantation, sondern auch nach dem erreichbaren Sportniveau im Sinne der Rückkehr zu präoperativer sportlicher Aktivität bedeutsam ist. Dieser Artikel soll einen Überblick über neuere Daten zu folgenden Fragen hinsichtlich Hüft-, Knie und Schulterendoprothetik geben: (1) Welche Auswirkungen hat sportliche Aktivität auf Endoprothesen? (2) Welches Sportniveau ist nach endoprothetischer Versorgung erreichbar? (3) Welche Empfehlungen können zu sportlicher Aktivität mit Endoprothese gegeben werden?

Methoden: PubMed basierte Literaturrecherche. Narrativer Review mit Fokus auf aktuellen Daten aus den Jahren 2010 - 2016.

Ergebnisse: Die allgemein bekannte Empfehlung zu Low-impact-Sportarten mit moderater Intensität wie Wandern, Schwimmen, Radfahren, Golf behält ihre Gültigkeit für sämtliche Prothesentypen an allen Gelenken. Hier ist unstrittig, dass die positiven die negativen Effekte überwiegen. Nach Implantation von HTEP und KTEP findet die Mehrheit der prä-OP aktiven Patienten nach 3-6 Monaten wieder zu sportlicher Aktivität zurück, wenn auch mit klarer Tendenz zu niedrigerer Intensität und einer Verschiebung von high impact hin zu low impact. Wichtige offene Fragen beziehen sich auf die Effekte von mit hoher Intensität betriebenen Low-impact-Sportarten, auf High-impact-Sportarten, auf sportartspezifische Wirkungen sowie auf prothesentypspezifische Möglichkeiten. Hier sind die Grenzen zwischen positiven und negativen Effekten von sportlicher Belastung unterschiedlicher Intensität erst beginnend untersucht. Neue Daten weisen darauf hin, dass allgemein eine höhere sportliche Leistungsfähigkeit erreichbar ist, als traditionell empfohlen wird. Frühe Ergebnisse unikondylärer Knieprothesen übertreffen die bikon-

\section{Abstract \\ $\nabla$}

Background: An increasing number of physically active patients not only need to know if they will basically be able to engage in sports after undergoing arthroplasty. They also would like to know whether or not they will be able to resume their preoperative activity levels. This article aims to provide an overview of recent data regarding the following questions on hip, knee and shoulder arthroplasty: (1) What is the impact of physical activity on an endoprosthesis? (2) What level of sports can be achieved after an arthroplasty procedure? (3) What types of sport are recommended for patients with an endoprosthesis?

Methods: PubMed-based review of the literature. Narrative review focusing on current data from the years 2010 to 2016.

Results: The commonly known recommendation to exercise low-impact sports such as hiking, swimming, cycling or golf at a moderate intensity remains valid for all types of prostheses in all joints. There is broad consensus that the benefits of these sports outweigh the negative effects. Having undergone total hip or knee arthroplasty, most patients with a high preoperative activity level return to sports after 3-6 months, albeit with a clear tendency to lower intensity and a shift from high-impact to low-impact sports. Some key questions have to be answered regarding the effects of low-impact sports that are exercised with high intensity, the effects resulting from high-impact sports, effects specific to different types of sport, and possibilities provided by different prosthesis types. In this context, a lot remains to be done to investigate the limits between positive and negative effects resulting from physical activity of varying intensity. New data suggests that generally a higher physical performance level may be achieved than has been traditionally recommended. Early results of unicondylar knee prostheses are far better than those 
dylärer Prothesen deutlich. Schulterendoprothesen zeigen entgegengesetzt zu Expertenempfehlungen die höchsten postoperativen Aktivitätslevel nach inverser und anatomischer TEP und die geringste Sportaktivität nach Hemiprothesen.

Schlussfolgerung: Bisherige Expertenempfehlungen und faktische Möglichkeiten des Sportniveaus nach Endoprothesenimplantation divergieren zum Teil erheblich. Es bleibt durch zukünftige Studien zu definieren, welches Sportniveau unter Verwendung moderner abriebarmer Gleitpaarungen und Prothesendesigns in welcher Sportart mit welcher Prothese ein positives Nutzen-Risiko-Verhältnis darstellt.

\section{Einleitung \\ $\nabla$}

Der Zusammenhang zwischen sportlicher Aktivität und Arthrose sowie sportlicher Aktivität und endoprothetischem Gelenkersatz ist komplex. Hinsichtlich der Pathogenese der Arthrose der unteren Extremität und sportlicher Belastung besteht eine Art Schwellenwertbeziehung. Einerseits hat regelmäßige körperliche Belastung mit moderater Intensität im Rahmen von Breiten- und Freizeitsport, insbesondere im Rahmen von Ausdauersportarten, protektive Effekte auf das Gelenkmilieu über verbesserte Nutrition des Knorpels und durch mechanische Entlastung aufgrund der Kräftigung der periartikulären Muskulatur. Andererseits besteht ein klarer Zusammenhang zwischen radiologisch diagnostizierbaren Hüft- und Kniegelenksarthrosezeichen und ehemaligem Sportniveau, mit höherer Prävalenz bei früheren Leistungsportlern im Vergleich zu Breitensportlern sowie nach Team-, Ball-, Kraftund Kontaktsportarten im Vergleich zu Ausdauersportarten, wobei letzterer Zusammenhang weniger stringent, aber auch bereits für Breitensportler gilt. Lefèvre-Colau et al. haben dies kürzlich in einer Übersichtsarbeit sehr ausführlich dargestellt [1]. Für alle Sportarten scheint die radiologisch bereits diagnostizierbare, aber klinisch noch asymptomatische Arthrose dabei interessanterweise vorwiegend durch Osteophytenbildung [2], aber oft noch ohne Gelenkspaltverschmälerung, gekennzeichnet zu sein. Die Entwicklung klinisch symptomatischer Arthrosen hingegen ist stark sportartspezifisch. Nicht die Belastungsdauer und -intensität per se, sondern vielmehr die kumulativen Gelenktraumata scheinen eine wesentliche Rolle zu spielen, mit deutlich vermehrter symptomatischer Arthrosebildung bei ehemaligen Leistungssportlern in Kontakt- und Ballsportarten, wie Fußball, Handball, Eishockey und Tennis und bei Kampfsportarten [3 - 8]. Die Prävalenz symptomatischer arthrotischer Gelenkschäden bei ehemaligen Leistungssportlern unterschiedlicher Disziplinen ist deutlich erhöht im Vergleich zur Allgemeinbevölkerung. Prozentuale Angaben zur sportartspezifischen Prävalenz existieren jedoch nicht, da viele Studien zu diesem Thema retrospektive Kohortenstudien sind und das sportartspezifische Verletzungsrisiko nicht immer adäquat berücksichtigt ist [9]. Allerdings ist nicht nur der grundsätzliche Zusammenhang zwischen Leistungssport und Arthroseprävalenz inzwischen unstrittig: erste Studien weisen auch auf eine erwartungsgemäß höhere Inzidenz von Knie- und Hüftendoprothesenimplantationen bei ehemaligen Leistungssportlern in Highimpact-Sportarten hin, wobei auch hier Gelenkverletzungen in der Vorgeschichte eine entscheidende Rolle zu spielen scheinen [10]. Die erfolgreiche Weiterentwicklung des endoprothetischen Gelenkersatzes an Hüfte, Knie und Schulter hat zu einer Ausweitung des Indikationsspektrums der Endoprothetik auch für jüngere, verhältnismäßig körperlich aktive Patienten geführt, zu denen achieved with bicondylar prostheses. In contrast to expert recommendations, shoulder endoprostheses show the highest postoperative activity levels after inverted arthroplasty, followed by anatomic arthroplasty, and the lowest activity level after the implantation of a hemiprosthesis.

Conclusion: There is a significant discrepancy between previous expert recommendations and the actual activity levels that may be achieved after the implantation of a joint prosthesis. Future studies have to define the sports level, the type of sports and the type of prosthesis that provide a positive benefit-risk ratio using state-of-the-art low-abrasion bearing surfaces and prosthesis designs.

insbesondere ehemalige (Leistungs-)Sportler gehören. Das Aktivitätsniveau der über 60-70jährigen Patienten steigt ebenfalls kontinuierlich an, sodass sich insgesamt zunehmend Patienten mit vergleichsweise hohem sportlichem Funktionsanspruch finden. Für eine wachsende Anzahl von Patienten steht inzwischen im Rahmen der Indikationsstellung zur Endoprothese nicht nur die Frage nach grundsätzlicher Sportfähigkeit, sondern auch nach dem erreichbaren Sportniveau im Sinne der Rückkehr zu präoperativer sportlicher Aktivität im Fokus [11].

Seit Jahrzehnten besteht für Hüft- und Knie-TEPs die allgemein bekannte Empfehlung zu Low-impact-Sportarten mit moderater Intensität wie Wandern, Schwimmen, Radfahren, Golf [12, 13]. Hier ist unstrittig, dass die positiven Effekte die negativen überwiegen.

Unsere heutigen Patienten konfrontieren uns jedoch zunehmend mit spezifischeren Bedürfnissen und Fragestellungen: Welchen Effekt haben Low-impact-Sportarten mit hoher Intensität? Ist High-impact-Sport grundsätzlich nicht zu empfehlen? Welche Sportart hat welche Auswirkung auf welche Prothese? Welches Sportniveau ist mit welchem Prothesentyp erreichbar?

Gutes Erwartungsmanagement erfordert eine realistische und fundierte Aufklärung seitens des Operateurs [14]. In der jüngeren Vergangenheit ist eine Vielzahl neuartiger Studien publiziert worden, die einige spezifische Subfragstellungen dieses Fragenkomplexes adressiert haben und zum Teil zu erstaunlichen Ergebnissen kommen.

Ziel dieses Artikels ist es daher, einen Überblick über die jüngere Datenlage mit dem Schwerpunkt auf Publikationen aus den Jahren 2010 - 2016 zu folgenden Fragen hinsichtlich der Hüft-, Knie und Schulterendoprothetik zu geben: (1) Welche Auswirkungen hat sportliche Aktivität auf die Endoprothese? (2) Welches Sportniveau ist nach endoprothetischer Versorgung erreichbar? (3) Welche Empfehlungen können zu sportlicher Aktivität mit Endoprothese gegeben werden?

\section{Methoden}

$\nabla$

Es handelt sich um eine nicht systematische Übersichtsarbeit. Die elektronische Datenbank PubMed (http://www.ncbi.nlm.nih.gov/ pubmed) diente als Basis für die Literaturrecherche. Thematisch relevante Artikel wurden nach Kombination folgender Schlagwörter gesucht: „sports AND arthroplasty“, „sports AND total joint replacement“, „return to sports AND arthroplasty", „physical activity AND implant survival", "physical activity AND aseptic loosening”, "guidelines AND sports AND arthroplasty", "recommendations AND sports AND joint replacement". Die Auswahl der zitierten Artikel erfolgte nach Ermessen der Autoren mit dem Ziel, die aktuelle 
Datenlage umfassend aber prägnant darzustellen sowie die jüngere Datenlage hervorzuheben. Es wurden nur englischsprachige Publikationen berücksichtigt. Der Fokus lag auf Schulter-, Knieund Hüftendoprothetik sowie den Publikationsjahren 20102016.

\section{Ergebnisse und Diskussion \\ $\nabla$}

\section{Einfluss sportlicher Aktivität auf Endoprothesen}

Zentraler Hintergrund aller Empfehlungen zu sportlicher Aktivität nach endoprothetischer Versorgung ist der direkte Einfluss mechanischer Belastung auf Abrieb, aseptische Prothesenlockerung, Luxationsrisiko und periprothetische Frakturen. Hierbei wurde traditionell in weitaus größerem Maß nach vermeintlicher Einschätzung, denn nach objektivierbarer Datenlage, geurteilt. Die herkömmliche Unterscheidung zwischen Low-impactund High-impact-Sportarten beruht auf angenommen, nicht systematisch und objektiv gemessenen, globalen Unterschieden an Intensität, Dauer und Frequenz der unmittelbaren mechanischen Belastungen auf das Kunstgelenk. Zu den Low-impactSportarten werden Walken, Wandern, Schwimmen, Fahrradfahren, Tanzen, Golf, Langlaufski und Tennis (Doppel) gezählt, während Tennis (Einzel), Squash, Fußball, Handball, Basketball, Volleyball, Ski alpin und Joggen als high impact gelten [15]. Zwar ist bekannt, dass bei unterschiedlichen Aktivitäten unterschiedliche Kräfte auf einzelne Gelenke wirken. Während beispielsweise beim Fahrradfahren Kräfte in der Größenordnung des 1,1fachen Körpergewichts auf das Knie wirken, sind es bei normalem Gehen das 2 - 3fache Körpergewicht und beim Tennis bis zum 4fachen des Körpergewichtes [16]. Es gibt jedoch bisher nur sehr wenige Messungen zur faktischen Belastung von Endoprothesen im Rahmen spezifischer Sportarten und nur sehr begrenzt konkrete Hinweise darauf, dass diese differenten Kräfte auch unter Verwendung moderner abriebarmer Gleitpaarungen zu negativen Konsequenzen für die Funktionalität und Standzeiten von Hüft-, Knie- und Schulterprothesen führen. Die naheliegende, schlichte Gleichung „mehr Aktivität = höheres Abrieb-, Lockerungs-, Luxations- und Frakturrisiko" gilt sicher in dieser Einfachheit nicht. Ebenso wie hinsichtlich des Zusammenhangs mit der Entstehung von Arthrose, gibt es auch hinsichtlich des Zusammenhangs zwischen sportlicher Belastung und deren Wirkung auf Endoprothesen eine Schwellenwertbeziehung. Einerseits fördert regelmäßige mechanische Belastung bis zu einem gewissen Grad die Knochenqualität, begünstigt damit die ossäre Fixation der Prothese und reduziert das Risiko periprothetischer Frakturen [17]. Zudem kann ein gut ausgebildeter periartikulärer Muskelapparat die unmittelbare Gelenkbelastung vermindern und im Rahmen allgemeiner körperlicher Aktivität das Sturzrisiko und damit die Gefahr periprothetischer Frakturen senken [18]. Andererseits besteht unzweifelhaft ein direkter Zusammenhang zwischen mechanischer Belastung und Gleitpaarungsabrieb mit dem wesentlichen Risiko PE-Abrieb bedingter Osteolysen und konsekutiver Implantatlockerung infolge mechanischer Überbelastung $[18,19]$.

Bezeichnenderweise sind jedoch die Grenzen zwischen nützlicher Belastung und schädlicher Überlastung einzelner Gelenke im Rahmen sportlicher Aktivität erstaunlich wenig konkret wissenschaftlich definiert. Es existiert wenig Literatur zu den Effekten von Sport auf Endoprothesen und evidenzbasierten De- finitionen nützlicher Belastungsbereiche in Abgrenzung von schädlichen Überbelastungen.

\section{Einfluss sportlicher Aktivität auf Hüftendoprothesen}

Polyethylen (PE) - Keramik Gleitpaarung

Ollivier et al. haben untersucht, ob „impact sport“ einen Einfluss auf die Langzeitergebnisse zementfreier Hüftendoprothesen (Keramik - konventionelles PE) hinsichtlich Luxation, linearem PEAbrieb und Revisionsraten hat. Nach einem mittleren Follow-up von 11 Jahren wurden sportlich sehr aktive Patienten (Selbstauskunft UCLA (University of California Los Angeles) score $>8, \mathrm{n}=70$ ) mit Patienten mit geringerem Aktivitätsniveau (UCLA score $<5$, $\mathrm{n}=140$ ) verglichen. In der sogenannten High-impact-Gruppe wurde mehr PE Abrieb (0,14 $\pm 0,06$ vs. 0,06 $\pm 0,04 \mathrm{~mm} / \mathrm{Jahr})$ und eine signifikant schlechtere Survival-Rate ( 80 vs. 93,5\% nach 15 Jahren, $\mathrm{p}<0,001$ ) beobachtet als in der Low-impact-Gruppe. Die Luxationsrate hingegen unterschied sich zwischen den Gruppen nicht [20].

Lübekke et al. haben ähnliche Ergebnisse aus einer retrospektiven Analyse von Hybrid TEPs 5 und 10 Jahre post implantationem berichtet [21]. Stratifiziert nach UCLA-Score in low activity $(\mathrm{n}=166)$ versus high activity $(\mathrm{n}=58)$ und adjustiert für Alter, Geschlecht, BMI und Pfanneninklinationswinkel ergab sich eine 3,6fach höhere Odds Ratio für femorale Osteolysen, nicht jedoch PE-Abrieb, sowie eine höhere Revisionsrate für aseptische Lockerung in der High-activity-Gruppe.

Interessanterweise waren in beiden Studien sowohl FunktionsScores als auch subjektive Zufriedenheit in der Gruppe mit hoher sportlicher Aktivität deutlich größer als in der Gruppe mit geringerer Aktivität. Beide Publikationen haben methodische Schwächen in der Erfassung des tatsächlichen Aktivitätslevels und lassen keine finalen Rückschlüsse auf den spezifischen Effekt einzelner Sportarten zu. Sie verdeutlichen aber das Dilemma, dass hohe Zufriedenheit aufgrund hoher Belastbarkeit und Funktionalität der Hüftprothese ab einer individuell noch nicht sicher vorhersagbaren Grenze wahrscheinlich zu einer Verkürzung der revisionsfreien Standzeit führt. Wichtig ist zu realisieren, dass beide Studien unter Verwendung konventioneller PE-Inlays erfolgten und noch nicht klar ist, ob dieser Zusammenhang auch für das heute in der Regel verwendete hoch vernetzte PE gilt.

\section{Metall-Metall (MoM) Oberflächenersatzprothesen (OFE)}

Eines der Argumente für den Einsatz von MoM-OFE ist die relativ gute Rekonstruktion der Hüftgelenksbiomechanik mit guter Propriozeption, die hohe Luxationssicherheit und niedrige Abriebraten, was besondere Vorteile für den sportlich aktiven Patienten bedeuten kann. Die kurzfristige Sportfähigkeit und die Rate an return to sports inklusive high impact ist nach MoM-OFE tatsächlich bestechend hoch [22 - 24]. Die einzige Studie jedoch, welche die Langzeitfolgen hoher Aktivität auf MoM-OFE untersucht hat, kam zu dem Ergebnis, dass auch hier durch High-impact-Sport höhere Revisionsraten bedingt werden: LeDuff und Amstutz haben an einem jungen und aktiven Patientenkollektiv $(n=445$, 74\% männlich, Durchschnittsalter bei Implantation 49 Jahre) nach einem Follow-up von 10 Jahren (6 - 14) festgestellt, dass Patienten mit hoher sportlicher Aktivität (Hip-Impact and CycleScore $>50$ ) über einen Beobachtungszeitraum von 1 - 5 Jahren ein 3,8fach höheres Revisionsrisiko hatten als Patienten mit niedriger Aktivität (11,2 vs. 4,6\% Revisionen nach 8 Jahren) [25]. 
Zusammenfassend gibt es sehr wenig systematisch erfasste Daten hinsichtlich des Effekts von sportlicher Aktivität auf die Komplikationsrate und Standzeit von Hüftendoprothesen. Die wenigen existierenden Studien lassen den Schluss zu, dass über einen längeren Zeitraum betriebener High-impact-Sport zwar mit hoher subjektiver Zufriedenheit, aber wahrscheinlich mit mittel- bis langfristig erhöhten Revisionsraten einhergeht.

\section{Einfluss sportlicher Aktivität auf Knieendoprothesen}

Einfluss auf Standzeit und Revisionsraten

Auch für Knieprothesen ist die Datenlage sehr überschaubar. Interessanterweise ist jedoch im Gegensatz zu Hüftendoprothesen nach Implantation von uni- und bikondylären Kniegelenksoberflächenersatzprothesen bisher keine Tendenz zu einem erhöhten Risiko für die Entstehung osteolytischer Herde, aseptischer Lockerungen und Revisionsraten in hoch aktiven Patienten im Vergleich zu weniger aktiven Patienten zu beobachten [26 - 28]. Für unikondyläre mediale Schlitten (Oxford III) haben Pietschmann et al. aus einer Kohorte von 131 Prothesen nach einem mittleren Follow-up von 4,2 Jahren keinen Unterschied in der Revisionsrate zwischen sportlich aktiven und inaktiven Patienten beobachtet [29]. Die Oxford-Gruppe hat ebenfalls für mediale unikondyläre Prothesen anhand von 1000 Implantationen stratifiziert nach Tegner Activity Score ( $\geq 5$ vs. $\leq 4$ ) und nach einem mittleren Follow-up von 6 Jahren keine erhöhte Komplikations- oder Revisionsrate in der Gruppe mit hoher körperlicher Aktivität $(n=115)$ im Vergleich zu den wenig aktiven Patienten $(n=885)$ beobachtet, sondern sogar eine signifikant bessere Survival-Rate $(p=0,025)$ in der Gruppe hoch aktiver Patienten [30].

Es bleibt zu konstatieren, dass derzeit keine überzeugenden Daten vorliegen, die auf eine erhöhte Revisionsrate von uni- oder bikondylären Knieprothesen infolge sportlicher Aktivität hinweisen.

Einfluss von Krafttraining auf die Kniegelenksmuskulatur bei liegender Prothese

Regelmäßiges Krafttraining (zweimal pro Woche über 13 Wochen) bei älteren Damen mit Kniegelenksarthrose und mit Kniegelenksprothesen kann geeignet sein, um ein Niveau der Muskelkraft, der Balance und der funktionellen Stabilität wieder herzustellen, welches dem eines Kontrollkollektivs ohne Kniegelenkspathologie vergleichbar ist [31, 32]. Petterson et al. haben beschrieben, dass nach Knie TEP durch ein progressives Krafttraining innerhalb der ersten 4 Wochen zunächst die Normalisierung der willkürlichen Aktivierung des M. quadriceps wesentlich zur Maximalkraft beiträgt, bevor dann im Verlauf die physiologische Bedeutung des Muskelquerschnitts für die maximale Kraftentfaltung wieder zum Tragen kommt (12 und 52 Wochen post-OP) [33].

Einfluss von alpinem Skisport auf Knieendoprothesen

Müller et al. haben nach Knieprothesenimplantation den Effekt eines 12-wöchigen Alpin-Ski-Trainings auf multiple Aspekte untersucht und in Form einer Serie von Supplements publiziert: Es wurden positive Effekte auf das allgemeine Wohlbefinden der Patienten, die Kniefunktion und das Schmerzempfinden, auf die Patellarsehne, auf das Gangbild einschließlich seitengleicher Gewichtsverteilung, auf den Muskelquerschnitt des M. quadriceps (M. rectus femoris), auf die Adaptation der Typ I Muskelfasern, auf den Glukosestoffwechsel sowie auf die Kontraktionskraft von Kniestreckern und -beugern berichtet [34-40]. Nach drei Saisons Alpin-Ski mit jeweils 80 Skitagen wurde in einer Gruppe von 16 Patienten keine erhöhte Rate von PE-Abrieb, Osteolysen oder Implantatlockerungen konstatiert [41].

\section{Effekt sportlicher Aktivität auf Schulterprothesen}

Unseres Wissens existieren keine publizierten Daten, die den direkten Einfluss von Sport auf Schulterprothesen untersucht haben.

\section{Zusammenfassung: Einfluss sportlicher Aktivität auf Endoprothesen}

Die Datenlage zum Einfluss spezifischer Sportarten auf einzelne Prothesentypen ist noch rudimentär, hier sind Chancen und Grenzen noch nicht klar definiert. Generell fehlt in der Literatur eine direkt vergleichende Betrachtung der positiven allgemeinen Effekte durch Sport in klarer Abgrenzung gegenüber den potenziell negativen Effekten auf die Prothesenstandzeit, also eine genauere Schwellenwertdefinition.

Für Low-impact-Sportarten gibt es keinerlei Hinweise in der Literatur, dass die Ausübung des Sports negative Konsequenzen für Endoprothesen haben könnte.

Hinsichtlich High-impact-Sportarten gibt es für Hüftprothesen erste Daten, die auf eine reduzierte Standzeit infolge hoher sportlicher Belastung hinweisen, für Knieprothesen ist ein solcher Zusammenhang nicht nachgewiesen, für Schulterprothesen fehlen jedwede spezifischen Studien.

Insgesamt ist die Datenlage aufgrund methodischer Schwächen hinsichtlich der Erfassung des Sportniveaus, kurzer Follow-upZeiträume und kleiner Patientenzahlen beschränkt aussagekräftig. Es bedarf weiterer Studien, um eine wissenschaftlich fundierte Grundlage für die Beratung des Patienten seitens des Operateurs, jenseits der Low-impact-Sportarten, zu gewährleisten. Insbesondere prospektiv kontrollierte Studien mit Langzeitbeobachtungen hinsichtlich potenziell negativer Konsequenzen infolge High-impact-, High-intensity-Sportarten nach Verwendung von hochvernetzem PE in der Gleitpaarung ebenso wie retrospektive Registeranalysen zu dieser Fragestellung könnten hier einen Beitrag leisten.

\section{Return to sports: Welches Sportniveau ist nach}

\section{Endoprothesenimplantation wieder erreichbar?}

Untersuchte Endpunkte in diesem Rahmen sind meist allgemeiner Natur, wie zum Beispiel die Quote der allgemeinen Rückkehr zu sportlicher Aktivität (Return to Sports), die Anzahl der ausgeübten Sportarten, deren Frequenz, Dauer und die einzelnen Disziplinen. Weiterhin wurden patientenbasierte Aktivitätsskalen wie unter anderem der University of California Los Angeles (UCLA) activity score oder der sehr ähnliche Tegner Score auf einer Skala von 0-10 verwendet [42]. Einige wenige Studien haben sportartspezifische Endpunkte analysiert.

\section{Hüftendoprothetik Schaftprothesen}

Nach Implantation einer Hüft-TEP liegt der Zeitraum bis zum sportlichen Wiedereinstieg zwischen ein und sechs Monaten [43 - 45]. Schmidutz et al. beschreiben in einer Studie mit Kurzschaftprothesen, dass $98 \%$ der Patienten, die bis kurz vor dem Eingriff sportlich aktiv waren, postoperativ ihre sportliche Betätigung mit einem UCLA Score von 7,6 wieder aufnehmen konnten. Postoperativ wurden im Schnitt 3,9 Sportarten im Vergleich zu 3,5 präoperativ ausgeübt. Innman et al. haben 11 Jahre nach zementfrei implantierten Standardschäften im Vergleich zur prä-OP noch asymptomatischen Phase eine Quote des sportlichen Wiedereinstieges von $89 \%$ beschrieben sowie eine Verbesserung des UCLA von 3,6 auf 6,2 bei unveränderter Anzahl (1,8 vs 1,7), Dauer und Frequenz der ausgeübten Sportarten [45]. Quoten des Return to Sports ohne genaue Differenzierung der Sportarten in der Größen- 
ordnung von 75 - 95\% entsprechen auch früheren Arbeiten in der Literatur [46, 47]. Allen Studien gemein ist ein postoperativ deutlicher Anstieg in der Ausübung von Low-impact-Sportarten wie Schwimmen und Fahrradfahren auf Kosten der High-impactSportarten wie Tennis und Fußball [43, 45, 47]. Ein aktuell publizierter direkter Vergleich zementfreier Kurz- und Langschaftprothesen hat hinsichtlich der Wiederaufnahme sportlicher Aktivitäten bei jungen Patienten (bei Implantation im Durchschnitt $<40$ Jahre) im mittelfristigen Verlauf von gut 4 Jahren Follow-up keine Unterschiede zwischen den Prothesentypen aufgedeckt [48]. Es gibt allgemein keine Daten, die auf Unterschiede hinsichtlich des Sportniveaus nach Implantation verschiedener Schaftprothesen hinweisen, wohl jedoch zwischen Schaftprothesen und Oberflächenersatzprothesen der Hüfte (s. u.).

\section{Hüftendoprothetik Oberflächenersatz (OFE)}

Die Vorteile hinsichtlich Geometrie, Propriozeption, Stabilität und Abrieb lassen den Oberflächenersatz am Hüftgelenk als bevorzugte Prothese bei jungen aktiven Patienten mit hohem Anspruch an den postoperativen Funktionserhalt erscheinen. Girard et al. haben in einer prospektiven Studie für 215 OFE-Prothesen nach einem mittleren Follow-up von 44 Monaten eine Wiederaufnahme sportlicher Aktivität von $98 \%$ der Patienten nach durchschnittlich 3 Monaten beschrieben, wobei Patienten mit prä-OP High-impact-Sportaktivität $(\mathrm{n}=55)$ auch zu $82 \%$ in den high impact zurückgekehrt sind. Der UCLA-Score zeigte einen Anstieg von 6,6 auf 9,1 [24]. Die gleiche Arbeitsgruppe hat anhand dieser Kohorte beschrieben, dass 40 präoperativ aktive Läufer nach durchschnittlich 16 Wochen zu $92 \%$ post-OP auf einem subjektiv als gut empfundenen Niveau im Laufsport wieder aktiv waren und hinsichtlich der Teilnahme an Wettkämpfen kein Unterschied von prä- zu post-OP bestand [23]. Ähnliche Ergebnisse beschreiben Sandiford et al. mit einem Return to Sports im Mittel 3 Monate post-OP nach OFE $(n=79)$ bei unveränderter sportlicher Intensität gegenüber prä-OP [49] sowie Banerjee et al. mit einer Return-toSports-Quote von $98 \%$ bei 152 OFE-Prothesen im Mittel 2 Jahre post-OP und einer gesteigerten allgemeinen Partizipation und Dauer der sportlichen Trainingseinheiten post-OP bei gleichzeitig deutlichem Shift von High-impact- hin zu Low-impact-Sportarten [50]. Bedigrew et al. beschrieben, dass 6 Monate post-OP nach OFEImplantation eine Zunahme der periprothetischen Knochendichte am proximalen Femur gegenüber prä-OP zu verzeichnen ist und leiten daraus indirekt ab, dass hinsichtlich der Frakturgefahr die Wiederaufnahme sportlicher Aktivität 6 Monate post-OP wahrscheinlich auch für High-impact-Sport sicher sein sollte [22].

\section{Hüftendoprothetik: spezielle Sportarten}

Jogging gilt als High-impact-Sportart. Abe et al. haben anhand einer Kohorte von 608 Patienten (532w, 85 m, mittleres Alter 62 Jahre) mit 804 Hüftprothesen die postoperative Laufsportaktivität im Mittel knapp 5 Jahre post-OP untersucht. Der Anteil der laufaktiven Patienten reduzierte sich von 5,4 auf 3,8\% bei durchschnittlich 4 Trainingseinheiten pro Woche von 29 Minuten Dauer über eine Strecke von 3,6 km. In der laufsportaktiven Gruppe wurden keine negativen Konsequenzen der hohen Aktivität wie Schmerz, erhöhte Metallionenkonzentration, Lockerung oder Osteolysen festgestellt [51]. Sämtliche bisher publizierte Studien weisen darauf hin, dass Golfsport nach Hüftprothesenimplantation gegenüber prä-OP uneingeschränkt und ohne relevanten Leistungsverlust oder erhöhte Risiken ausführbar ist, wobei der vollen bewussten und unterbewussten Belastbarkeit der operierten Extremität für das Erreichen des ehemaligen Niveaus eine wichtige Rolle zuzukommen scheint [52 - 54]. Die häufig formulierte Empfehlung gegen Tennis als Einzelsportart ist unseres Wissens nicht durch Studien belegbar. Existierende Studien zu Tennis haben keinen negativen Effekt auf Hüftendoprothesen nachgewiesen [55].

\section{Knieendoprothetik: allgemeine Sportfähigkeit}

Der unikondyläre Oberflächenersatz (UKA) hat im Vergleich zum bikondylären Ersatz (TKA) aus funktioneller Sicht den großen Vorteil des Erhalts beider Kreuzbänder, besserer Propriozeption und einer natürlicheren Kinematik des Kniegelenks. In einer systematischen Übersichtsarbeit haben Witjes et al. 18 Originalarbeiten hinsichtlich der postoperativen sportlichen Fähigkeiten nach UKA und TKA gegenübergestellt. Der Zeitrahmen der Rückkehr zu sportlicher Aktivität betrug im Mittel 12 (UKA) vs. 13 (TKA) Wochen. Die Quote der Rückkehr zu sportlicher Aktivität betrug für UKA bis zu > 100\% der prä-OP sportlich aktiven Patienten, für TKA bis zu 89\%. Die Anzahl der ausgeübten Sportarten war nach UKA mit 1,1-4,6 ebenfalls deutlich höher als nach TKA $(0,2-1,0)$. Bei der Interpretation dieser Daten sollte man berücksichtigen, dass es sich nicht um prospektiv randomisierte Studien mit vergleichbarem Ausgangskollektiv handelt, sondern um Einzelstudien mit unter Umständen erheblichen Variationen der präoperativen Pathologie und des Fitnesszustands der Kollektive. Ho et al. haben in einer frisch publizierten Arbeit ein Kollektiv mit prä-OP vergleichbaren klinischen und radiologischen Befunden hinsichtlich der Sportaktivität 4 Jahre post-OP analysiert, nachdem $\mathrm{n}=33$ mittels UKA und $\mathrm{n}=39$ mittels TKA versorgt worden waren und dabei zwar bessere Funktions-Scores für UKA, jedoch keinen Unterschied in der Rate des Return to Sports festgestellt [56].

Es bleibt aktuell unklar, ob die allgemein höhere sportliche Aktivität nach UKA auf die Unterschiede der Prothesenfunktionalität (UKA vs. TKA unter der Annahme vergleichbarer Ausgangsbefunde) oder auf Unterschiede der Ausgangsbefunde zum Zeitpunkt der Indikationsstellung zurückzuführen sind.

Ähnlich wie in der Hüftendoprothetik zeigt die Literatur sowohl nach UKA und TKA eine deutliche Verschiebung von High-impact-Sportarten zu Low-impact-Sportarten, wobei dieser Trend nach TKA stärker ausgeprägt ist [13]. Eine aktuelle Arbeit von Vielgut et al. hat die Sportpartizipation von 236 Patienten im Langzeitverlauf nach einem mittleren Follow-up von 14,9 Jahren nach TKA beschrieben [57]. Immerhin $71 \%$ der Patienten waren 15 Jahre post-OP noch sportlich aktiv, jedoch war auch hier eine deutliche Verschiebung hin zum low impact zu verzeichnen: von den Patienten, die prä-OP High-impact-Sport betrieben hatten, waren es post-OP nur noch $16 \%$, von denen mit Intermediate-impact-Sport noch $44 \%$, von denen mit Low-impact-Sport noch $71 \%$. Diese differenzierte Unterscheidung inklusive Medium/Intermediate-impact-Sports gewinnt in der Literatur zur Knieendoprothetik zunehmend an Akzeptanz. Mayr et al. haben $n=81$ ältere Patienten (> 60 Jahre) im Mittel 6,5 Jahre post-OP mit TKA nachuntersucht und hohe sportliche Aktivität konstatiert [58]: die Patienten waren im Wochenmittel in 3-4 Trainingseinheiten über 5 Stunden sportlich aktiv, wobei 25\% High-impact-, $47 \%$ Medium- und $51 \%$ Low-impact-Sportarten betrieben. Es ist seit weit über 10 Jahren bekannt, dass Patienten, die prä-OP in Highimpact-Sportarten aktiv waren durchaus in der Lage sind, diesen Sport auch post-TKA mit hoher subjektiver Zufriedenheit und ohne negative Konsequenzen im mittelfristigen Verlauf auszuüben $[59,60]$. Wenn man die Frage umkehrt und analysiert, warum Patienten nach Knieprothesenimplantation keinen Sport 
treiben, so wird evident, dass die Hinderungsgründe wahrscheinlich ganz überwiegend nicht mit dem operierten Knie zusammenhängen, sondern eher mit dem Allgemeinzustand der Patienten in Zusammenhang stehen [61].

\section{Knieendoprothetik: spezielle Sportarten}

Sämtliche bisher publizierten Arbeiten die sich speziell auf Golf beziehen, kommen zu dem Schluss, dass Golfsport nach Knieprothesenimplantation gegenüber prä-OP uneingeschränkt oder besser möglich ist, wobei der Schmerzreduktion hierbei eine tragende Rolle zukommt [52, 62]. Auch Tennis, sowohl als Einzel als auch als Doppel, ist bei prä-OP auf hohem Niveau trainierten Tennisspielern auch post-TKA mit hoher subjektiver Zufriedenheit bei 3 - 4 Trainingseinheiten pro Woche durchführbar [55, 63].

\section{Schulterendoprothetik: allgemeine Sportfähigkeit}

Der Wunsch nach hoher Belastbarkeit einer Schulterprothese, zum Beispiel durch eine körperlich beanspruchende berufliche Tätigkeit oder durch Sport, hat traditionell als ein Argument für die Implantation von Hemiprothesen in Abgrenzung zu Totalendoprothesen gegolten [64]. Interessanterweise zeichnet sich in den aktuellen Publikationen der Jahre 2010-2016 ab, dass die Sportfähigkeit von Patienten mit Hemiprothesen schlechter ist als von Patienten mit anatomischen und inversen Totalendoprothesen. Unabhängig vom Prothesentyp kann man davon ausgehen, 5-8 Monate post-OP wieder sportfähig zu sein [65]. Die Rückkehrquote liegt dabei allerdings für die Hemiarthroplastik mit $60-70 \%$ erstaunlicherweise deutlich niedriger als bei der inversen TEP (etwa $85 \%$ ), die besten Ergebnisse werden mit 90 - 100\% nach anatomischer TEP erzielt [64-66]. Garcia et al. publizierten im direkten Vergleich zwischen Hemiprothesen $(n=40)$ und anatomischer TEP $(n=40)$ im mittleren Follow-up von gut 5 Jahren eine Rückkehrquote zu High-impact-Sportarten von $89 \%$ für anatomische TEPs, hingegen von nur $63 \%$ für Hemiprothesen sowie eine signifikant höhere Partizipation an Sportarten mit aktiver Beteiligung der oberen Extremität wie beispielsweise Schwimmen oder Golfen nach TEP (87 vs. $48 \%$ ) [64]. Auch ein direkter Vergleich zwischen Hemiarthroplastik ( $n=71,66$ Jahre) und inverser TEP $(n=102,72$ Jahre) deckte eine deutliche Unterlegenheit der Hemiprothesen auf [65]: Die Rückkehrrate nach inverser Prothese betrug 86 versus $67 \%$ mit Hemiprothese, die subjektive Zufriedenheit hinsichtlich der Sportfähigkeit war bei den Patienten mit inverser Prothese signifikant höher und die Rate von Restbeschwerden signifikant niedriger (63 vs $29 \%, \mathrm{P}<0,0001$ ). Wang et al. haben kürzlich anhand einer Kohorte von 51 Patienten mit Inverser Prothese (74 Jahre bei Implantation) und einem mittleren Follow-up von etwa 2,5 Jahren bestätigt, dass über 70\% der Patienten mit inverser Prothese zu anspruchsvoller sportlicher Aktivität zurückkehren können und die isokinetische Muskelkraft insbesondere in Flexion und Innenrotation dabei ein wesentlicher Faktor bezüglich des erreichten Sportniveaus ist [67]. Eine Reihe weiterer Studien aus den vergangenen zwei Jahren bestätigt die eher unerwartet gute Sportfähigkeit mit inversen Prothesen im kurz- bis mittelfristigen Verlauf mit bis zu über $90 \%$ allgemeiner Rückkehrquote und 20 - 25\% Rückkehrquote sogar in High-impact-Sportarten binnen etwa 6 Monaten post-OP ohne erhöhte Komplikationsraten [68-70]. Für anatomische TEPs auf dem Boden einer primären Omarthrose konnten Bülhoff et al. anhand einer Kohorte von $n=154$ Patienten, stratifiziert nach präoperativer sportlicher Aktivität zeigen, dass $100 \%$ der prä-OP aktiven Patienten $(n=105)$ auch post-OP wie- der sportlich aktiv waren, während prä-OP inaktive Patienten $(\mathrm{n}=49)$ auch post-OP sehr wahrscheinlich inaktiv bleiben [71]. Ähnliche Ergebnisse haben Schumann et al. anhand 100 konsekutiver anatomischer TEP-Implantationen, darunter 55 Sportler, gefunden, wobei nach knapp 3 Jahren bei prä-OP aktiven Patienten, von knapp 90\% das ehemalige Sportniveau nahezu wieder erreicht wurde [66]. Zu den favorisierten Sportarten nach anatomischer TEP gehören Schwimmen, Fitness, Tennis und Golf [66, 72].

\section{Schulterendoprothetik: spezielle Sportarten}

Eine Befragung von 367 Patienten im Mittel 3,2 Jahre nach anatomischer TEP-Implantation hat ergeben, dass 8 Monate post-OP die Rückkehr zum Golfsport mit signifikant weniger Schmerz, verbesserter Schlagweite und reduziertem Handicap verbunden ist [73].

\section{Welche Empfehlungen können zu sportlicher Aktivität} mit Endoprothese gegeben werden?

Die aktuellen Empfehlungen für Sport mit Endoprothesen stützen sich im Wesentlichen auf Expertenmeinungen aus Umfragen und entsprechen nicht evidenzbasierten Leitlinien. Es ist zu erwarten, dass sich dies in Zukunft ändern wird, unter anderem aufgrund der oben zitierten aktuellen Literatur und Entwicklungen.

So gibt es hinsichtlich der Hüft- und Kniegelenksendoprothetik trotz beginnend distinkter Datenlage für beide Gelenke noch keine klar separaten Empfehlungen, die der Datenlage entsprechen. Es besteht allgemein große Übereinkunft hinsichtlich folgender Punkte: nach einem postoperativen Zeitintervall von 3-6 Monaten werden Low-impact-Sportarten wie Walking, Fahrradfahren, Schwimmen und Golfen empfohlen, während von High-impact-Sportarten wie Joggen und Fußball abgeraten wird. Disziplinen wie Tennis und Skifahren werden meist differenzierter betrachtet (Tennis: Einzel vs. Doppel, Skifahren: Piste vs. Gelände, $\bigcirc$ Tab. 1, 2). Empfehlungen für Knieendoprothesen sind im Allgemeinen deutlich restriktiver als für Hüftendoprothesen [12, 74] ohne dass dieser Unterschied durch Daten begründet werden könnte. Es besteht Konsens zu liberaleren Empfehlungen, wenn die Sportart bereits vor dem Eingriff ausgeübt wurde $[74,75]$ ( $\diamond$ Tab. 1, 2). Interessanterweise korreliert die persönliche Erfahrung eines Operateurs (Anzahl der durchgeführten Eingriffe) mit der Tendenz zu liberaleren postoperativen Empfehlungen mit wachsender Erfahrung [12]. Generell sind die Empfehlungen von Hüft- und Kniechirurgen in den letzten 15-20 Jahren sukzessive liberaler geworden. Darüber hinaus bestehen deutliche regionale Unterschiede, was den subjektiven Charakter der Empfehlungen unterstreicht. Aus diesen beiden Trends sind die zum Teil beträchtlichen Abweichungen in den Ergebnissen aus Umfragen unter Experten zu erklären, wie sie in 0 Tab. 1, 2 nach Befragung nordamerikanischer und dänischer Orthopäden, publiziert in den Jahren 2009 und 2014 gegenübergestellt sind $[12,74]$.

Für Schulterendoprothesen bleiben die ausgesprochenen Empfehlungen bisher sehr allgemeiner Natur. Magnussen et al. haben 2011 eine Umfrage unter Experten der American Shoulder and Elbow Surgeons (ASES) und der European Society for Surgery of the Shoulder and Elbow (SECEC) durchgeführt an der 47 amerikanische und 52 europäische Kollegen teilnahmen [76]. Hier wurde mehrheitlich die Ausübung von Low-impact-Sportarten wie Walking, Fahrradfahren und Tanzen bei allen Prothesentypen nach 5-7 Monaten empfohlen. Low-impact-Sportarten, die 
Tab. 1 Empfehlungen zu sportlicher Aktivität nach Hüftendoprothese. Auszug aus Expertenumfragen unter dänischen (Laursen et al. 2014) und amerikanischen (Swanson et al. 2009) Chirurgen.

\begin{tabular}{|c|c|c|c|c|c|c|}
\hline & \multicolumn{2}{|l|}{ erlaubt \% } & \multirow{2}{*}{$\begin{array}{l}\text { gelegentlich } \\
\text { erlaubt \% } \\
\text { Swanson et al. } \\
\text { (2009) }\end{array}$} & \multirow{2}{*}{$\begin{array}{l}\text { erlaubt mit } \\
\text { Erfahrung \% } \\
\text { Laursen et al. } \\
\text { (2014) }\end{array}$} & \multicolumn{2}{|c|}{ nicht empfohlen \% } \\
\hline & $\begin{array}{l}\text { Swanson et al. } \\
\text { (2009) }\end{array}$ & $\begin{array}{l}\text { Laursen et al. } \\
\text { (2014) }\end{array}$ & & & $\begin{array}{l}\text { Swanson et al. } \\
\text { (2009) }\end{array}$ & $\begin{array}{l}\text { Laursen et al. } \\
\text { (2014) }\end{array}$ \\
\hline Walking (ebener Untergrund) & 99,3 & & 0,7 & & 0,0 & \\
\hline Joggen & 7,3 & 68,0 & 20,9 & 4,0 & 71,5 & 28,0 \\
\hline Schwimmen & 99,3 & 92,0 & 0,0 & 8,0 & 0,7 & 0,0 \\
\hline \multicolumn{7}{|l|}{ Fahrradfahren } \\
\hline - ebener Untergrund & 97,8 & 96,9 & 2,2 & 4,0 & 0,0 & 0,0 \\
\hline - Mountainbiking & 31,6 & 60,0 & 32,4 & 24,0 & 35,3 & 16,0 \\
\hline Wandern & & 88,0 & & 12,0 & & 0,0 \\
\hline Klettern & 53,7 & & 25,2 & & 20,6 & \\
\hline Golf & 99,3 & 92,0 & 0,0 & 8,0 & 0,7 & 0,0 \\
\hline \multicolumn{7}{|l|}{ Tennis } \\
\hline - Einzel & 17,4 & 56,0 & 32,4 & 40,0 & 50,0 & 4,0 \\
\hline - Doppel & 70,1 & & 26,6 & & 2,9 & \\
\hline Fußball & & 16,0 & & 16,0 & & 68,0 \\
\hline \multicolumn{7}{|l|}{ Skifahren } \\
\hline - Piste & 44,9 & & 39,6 & & 14,7 & \\
\hline - Skirennen & & 32,0 & & 48,0 & & 20,0 \\
\hline - Gelände & 5,9 & & 10,1 & & 83,7 & \\
\hline
\end{tabular}

Tab.2 Empfehlungen zu sportlicher Aktivität nach Knieendoprothese. Auszug aus Expertenumfragen unter dänischen (Laursen et al. 2014) und amerikanischen (Swanson et al. 2009) Chirurgen.

\begin{tabular}{|c|c|c|c|c|c|c|}
\hline & \multicolumn{2}{|l|}{ erlaubt \% } & \multirow{2}{*}{$\begin{array}{l}\text { gelegentlich } \\
\text { erlaubt \% } \\
\text { Swanson et al. } \\
\text { (2009) }\end{array}$} & \multirow{2}{*}{$\begin{array}{l}\text { erlaubt mit } \\
\text { Erfahrung \% } \\
\text { Laursen et al. } \\
\text { (2014) }\end{array}$} & \multicolumn{2}{|c|}{ nicht empfohlen \% } \\
\hline & $\begin{array}{l}\text { Swanson et al. } \\
\text { (2009) }\end{array}$ & $\begin{array}{l}\text { Laursen et al. } \\
\text { (2014) }\end{array}$ & & & $\begin{array}{l}\text { Swanson et al. } \\
\text { (2009) }\end{array}$ & $\begin{array}{l}\text { Laursen et al. } \\
\text { (2014) }\end{array}$ \\
\hline Walking (ebener Untergrund) & 99,3 & & 0,7 & & 0,0 & \\
\hline Joggen & 4,3 & 33,0 & 20,1 & 10,0 & 75,4 & 57,0 \\
\hline Schwimmen & 99,3 & 100,0 & 0,0 & 0,0 & 0,7 & 0,0 \\
\hline \multicolumn{7}{|l|}{ Fahrradfahren } \\
\hline - ebener Untergrund & 96,4 & 100,0 & 3,6 & 0,0 & 0,0 & 0,0 \\
\hline - Mountainbiking & 27,0 & 57,0 & 36,7 & 19,0 & 35,8 & 19,0 \\
\hline Wandern & & 81,0 & & 14,0 & & 5,0 \\
\hline Klettern & 55,1 & & 26,6 & & 17,6 & \\
\hline Golf & 97,8 & 95,0 & 1,4 & 5,0 & 0,7 & 0,0 \\
\hline \multicolumn{7}{|l|}{ Tennis } \\
\hline - Einzel & 10,9 & 43,0 & 28,1 & 43,0 & 60,6 & 14,0 \\
\hline - Doppel & 65,7 & & 28,8 & & 5,1 & \\
\hline Fußball & & 5,0 & & 10,0 & & 86,0 \\
\hline \multicolumn{7}{|l|}{ Skifahren } \\
\hline - Piste & 43,8 & & 39,6 & & 16,1 & \\
\hline - Skirennen & & 33,0 & & 48,0 & & 19,0 \\
\hline - Gelände & 3,7 & & 10,1 & & 85,9 & \\
\hline
\end{tabular}

den Einsatz der Arme erfordern, wie Golf und Schwimmen wurden zwar nach Hemiarthroplastik und anatomischer TEP empfohlen, bei inverser TEP jedoch nur sehr restriktiv unter der Voraussetzung vorangehender Erfahrung. High-impact-Sportarten wie Tennis, Skifahren und Fußball wurden für Hemiprothesen und anatomische TEPs mit der Einschränkung sportlicher Erfahrung empfohlen, jedoch für inverse Prothesen abgelehnt. Es zeigte sich allgemein eine deutlich zunehmende Restriktivität der Empfehlungen von der Hemiarthroplastik über die anatomische TEP zur inversen Endoprothese [76]. Eine 2012 von Golant et al. publizierte Umfrage unter ASES Mitgliedern bestätigte den Ein- druck, dass in den Augen der Operateure tendenziell ein Glenoidersatz (anatomisch oder invers) sowie Kontaktsportarten Hinderungsgründe gegenüber Wiederaufnahme sportlicher Aktivität post-OP darstellen sollten, sodass für Hemiprothesen die liberalsten Empfehlungen ausgesprochen werden [77].

Es wird deutlich, dass die existierenden Expertenempfehlungen mehr auf subjektiver Einschätzung des Risikos als auf objektiver Beobachtung des tatsächlichen Risikos und der faktischen Sportfähigkeit beruhen. Es fehlen aktuell evidenzbasierte Leitlinien, die für den sportlich ambitionierten Patienten eine fundierte 
Stütze bei der Wiederaufnahme sportlicher Aktivität nach endoprothetischer Versorgung darstellen könten.

\section{Schlussfolgerung}

Eine große Mehrheit der präoperativ körperlich aktiven Patienten ist nach dem operativen Eingriff wieder in nahezu gleichem Ausmaß in der Lage, sportlich aktiv zu sein. Generell ist ein Trend hin zu weniger Intensität und Impact der betriebenen Sportarten zu beobachten, wobei unklar ist, ob dieser Umstand eine Folge der eher restriktiven Empfehlungen oder der subjektiv empfundenen Möglichkeiten ist. Teilweise wird post-OP sogar ein höheres Aktivitätslevel erreicht, wobei der präoperative allgemeine und sportartspezifische Fitnesszustand sowie das Lebensalter für das post-OP erreichte Niveau eine entscheidende Rolle spielt. Ob sich eine intensive sportliche Belastung im Rahmen des individuell Möglichen langfristig im Sinne einer erhöhten Komplikations- oder Revisionsrate auswirkt, ist für Hüft-, Knie- und Schulterprothesen nur beginnend und noch nicht hinreichend geklärt. Empfehlungen der Fachgesellschaften und Expertengruppen sind allgemein restriktiver als das allgemein faktisch erreichbare Sportniveau. Die letzten 15 - 20 Jahre zeigen einen Trend hin zu liberaleren Empfehlungen.

Unserer Einschätzung nach sollte die Empfehlung des Operateurs im Einzelfall primär auf der individuellen Konstitution und den Bedürfnissen des Patienten unter Aufklärung über die wenigen bekannten und vorwiegend unbekannten Risiken basieren. Dabei ist es für ambitionierte Freizeitsportler wahrscheinlich hilfreich, die Priorisierung der sportbezogenen Lebensqualität für den Patienten einerseits, und die möglicherweise dadurch kompromittierte postoperative revisionsfreie Standzeit des Implantates andererseits klar zu formulieren. Leistungssportler aus dem Bereich von Highimpact-Sportarten beispielsweise, die ungeachtet der einschränkenden Empfehlung des Operateurs postoperativ wieder ihre angestrebte Sportart ausübten, konnten vielversprechende sportliche Niveaus erreichen, wodurch sich die potenzielle Bandbreite postoperativ möglicher Belastungen abzeichnet $[63,78]$.

Es besteht somit weiterhin ein großes, weitestgehend ungeklärtes Spannungsfeld in der Wechselwirkung zwischen faktischen Möglichkeiten sportlicher Aktivität, subjektiv gebotener Vorsicht und biomechanischen Grenzen der Implantate. Prospektive klinische Langzeitstudien und Registerstudien werden helfen, diese Grenzen zukünftig noch spezifischer zu definieren.

\section{Interessenkonflikt: Nein}

\section{Literatur}

1 Lefevre-Colau MM, Nguyen C, Haddad R et al. Is physical activity, practiced as recommended for health benefit, a risk factor for osteoarthritis? Ann Phys Rehabil Med 2016; 59: 196-206

2 Urquhart DM, Tobing JF, Hanna FS et al. What is the effect of physical activity on the knee joint? A systematic review. Medicine and science in sports and exercise 2011; 43: 432-442

3 Kuijt MT, Inklaar H, Gouttebarge V et al. Knee and ankle osteoarthritis in former elite soccer players: a systematic review of the recent literature. J Sci Med Sport 2012; 15: 480-487

4 Gouttebarge V, Inklaar H, Frings-Dresen MH. Risk and consequences of osteoarthritis after a professional football career: a systematic review of the recent literature. The Journal of sports medicine and physical fitness 2014; 54: 494-504

5 Molloy MG, Molloy CB. Contact sport and osteoarthritis. Br J Sports Med 2011; 45: 275-277
6 Richmond SA, Fukuchi RK, Ezzat A et al. Are joint injury, sport activity, physical activity, obesity, or occupational activities predictors for osteoarthritis? A systematic review. The Journal of orthopaedic and sports physical therapy 2013; 43: b515-b519

7 Driban JB, Eaton CB, Lo GH et al. Association of knee injuries with accelerated knee osteoarthritis progression: data from the Osteoarthritis Initiative. Arthritis Care Res (Hoboken) 2014; 66: 1673-1679

8 Silverwood V, Blagojevic-Bucknall M, Jinks C et al. Current evidence on risk factors for knee osteoarthritis in older adults: a systematic review and meta-analysis. Osteoarthritis Cartilage 2015; 23: 507-515

9 Gouttebarge V, Inklaar H, Backx F et al. Prevalence of osteoarthritis in former elite athletes: a systematic overview of the recent literature. Rheumatol Int 2015; 35: 405 - 418

10 Tveit $M$, Rosengren $B E$, Nilsson JA et al. Former male elite athletes have a higher prevalence of osteoarthritis and arthroplasty in the hip and knee than expected. Am J Sports Med 2012; 40: 527-533

11 Healy WL, Sharma S, Schwartz B et al. Athletic activity after total joint arthroplasty. J Bone Joint Surg Am 2008; 90: 2245 - 2252

12 Swanson EA, Schmalzried TP, Dorey FJ. Activity recommendations after total hip and knee arthroplasty: a survey of the American Association for Hip and Knee Surgeons. The Journal of arthroplasty 2009; 24: 120 126

13 Witjes S, Gouttebarge V, Kuijer PP et al. Return to Sports and Physical Activity After Total and Unicondylar Knee Arthroplasty: A Systematic Review and Meta-Analysis. Sports medicine (Auckland, NZ) 2016; 46: 269-292

14 Noble PC, Conditt MA, Cook KF et al. The John Insall Award: Patient expectations affect satisfaction with total knee arthroplasty. Clin Orthop Relat Res 2006; 452: 35-43

15 Vannini F, Spalding T, Andriolo L et al. Sport and early osteoarthritis: the role of sport in aetiology, progression and treatment of knee osteoarthritis. Knee surgery, sports traumatology, arthroscopy: official journal of the ESSKA 2016; 24: 1786-1796

16 D'Lima DD, Steklov N, Patil S et al. The Mark Coventry Award: in vivo knee forces during recreation and exercise after knee arthroplasty. Clin Orthop Relat Res 2008; 466: 2605-2611

17 Rosenbaum TG, Bloebaum RD, Ashrafi $S$ et al. Ambulatory activities maintain cortical bone after total hip arthroplasty. Clin Orthop Relat Res 2006; 450: 129-137

18 Golant A, Christoforou DC, Slover JD et al. Athletic participation after hip and knee arthroplasty. Bull NYU Hosp Jt Dis 2010; 68: 76-83

19 Gallo J, Goodman SB, Konttinen YT et al. Osteolysis around total knee arthroplasty: a review of pathogenetic mechanisms. Acta Biomater 2013; 9: 8046-8058

20 Ollivier M, Frey S, Parratte $S$ et al. Does impact sport activity influence total hip arthroplasty durability? Clin Orthop Relat Res 2012; 470: 3060 - 3066

21 Lubbeke A, Garavaglia G, Barea C et al. Influence of patient activity on femoral osteolysis at five and ten years following hybrid total hip replacement. J Bone Joint Surg Br 2011; 93: 456-463

22 Bedigrew KM, Ruh EL, Zhang Q et al. 2011 Marshall Urist Young Investigator Award: when to release patients to high-impact activities after hip resurfacing. Clin Orthop Relat Res 2012; 470: 299-306

23 Fouilleron $N$, Wavreille $G$, Endjah $N$ et al. Running activity after hip resurfacing arthroplasty: a prospective study. Am J Sports Med 2012; 40: 889-894

24 Girard J, Miletic B, Deny A et al. Can patients return to high-impact physical activities after hip resurfacing? A prospective study. Int Orthop 2013; 37: 1019-1024

25 Le Duff MJ, Amstutz HC. The relationship of sporting activity and implant survivorship after hip resurfacing. J Bone Joint Surg Am 2012; 94: $911-918$

26 Cherian JJ, Jauregui JJ, Banerjee S et al. What Host Factors Affect Aseptic Loosening After THA and TKA? Clin Orthop Relat Res 2015; 473: $2700-2709$

27 Jones DL, Cauley JA, Kriska AM et al. Physical activity and risk of revision total knee arthroplasty in individuals with knee osteoarthritis: a matched case-control study. J Rheumatol 2004; 31: 1384-1390

28 Mont MA, Marker DR, Seyler TM et al. Knee arthroplasties have similar results in high- and low-activity patients. Clin Orthop Relat Res 2007; 460: $165-173$

29 Pietschmann MF, Wohlleb L, Weber P et al. Sports activities after medial unicompartmental knee arthroplasty Oxford III-what can we expect? Int Orthop 2013; 37: 31 - 37 
30 Ali AM, Pandit $H$, Liddle AD et al. Does activity affect the outcome of the Oxford unicompartmental knee replacement? Knee 2016; 23: 327 330

31 Ciolac EG, Silva JM, Greve JM. Effects of resistance training in older women with knee osteoarthritis and total knee arthroplasty. Clinics (Sao Paulo) 2015; 70: 7-13

32 Ciolac EG, Greve JM. Muscle strength and exercise intensity adaptation to resistance training in older women with knee osteoarthritis and total knee arthroplasty. Clinics (Sao Paulo) 2011; 66: 2079-2084

33 Petterson SC, Barrance P, Marmon AR et al. Time course of quad strength, area, and activation after knee arthroplasty and strength training. Medicine and science in sports and exercise 2011; 43: 225 231

34 Wurth S, Finkenzeller T, Potzelsberger B et al. Alpine Skiing With total knee ArthroPlasty (ASWAP): physical activity, knee function, pain, exertion, and well-being. Scand J Med Sci Sports 2015; 25 (Suppl 2): $74-81$

35 Kosters A, Rieder F, Wiesinger HP et al. Alpine Skiing With total knee ArthroPlasty (ASWAP): effect on tendon properties. Scand J Med Sci Sports 2015; 25 (Suppl 2): $67-73$

36 Potzelsberger B, Stoggl T, Scheiber P et al. Alpine Skiing With total knee ArthroPlasty (ASWAP): symmetric loading during skiing. Scand J Med Sci Sports 2015; 25 (Suppl 2): 60-66

37 Potzelsberger B, Lindinger SJ, Stoggl T et al. Alpine Skiing With total knee ArthroPlasty (ASWAP): effects on gait asymmetries. Scand J Med Sci Sports 2015; 25 (Suppl 2): 49-59

38 Kristensen $M$, Potzelsberger B, Scheiber P et al. Alpine Skiing With total knee ArthroPlasty (ASWAP): metabolism, inflammation, and skeletal muscle fiber characteristics. Scand J Med Sci Sports 2015; 25 (Suppl 2): $40-48$

39 Rieder F, Kosters A, Wiesinger HP et al. Alpine Skiing With total knee ArthroPlasty (ASWAP): muscular adaptations. Scand J Med Sci Sports 2015; 25 (Suppl 2): 26-32

40 Potzelsberger B, Stoggl T, Lindinger SJ et al. Alpine Skiing With total knee ArthroPlasty (ASWAP): effects on strength and cardiorespiratory fitness. Scand J Med Sci Sports 2015; 25 (Suppl 2): 16-25

41 Hofstaedter T, Fink C, Dorn U et al. Alpine Skiing With total knee ArthroPlasty (ASWAP): clinical and radiographic outcomes. Scand J Med Sci Sports 2015; 25 (Suppl 2): 10-15

42 Naal FD, Impellizzeri FM, Leunig $M$. Which is the best activity rating scale for patients undergoing total joint arthroplasty? Clin Orthop Relat Res 2009; 467: 958 -965

43 Schmidutz F, Grote S, Pietschmann $M$ et al. Sports activity after shortstem hip arthroplasty. Am J Sports Med 2012; 40: 425 - 432

44 Cowie JG, Turnbull GS, Ker AM et al. Return to work and sports after total hip replacement. Arch Orthop Trauma Surg 2013; 133: 695 - 700

45 Innmann MM, Weiss S, Andreas F et al. Sports and physical activity after cementless total hip arthroplasty with a minimum follow-up of 10 years. Scand J Med Sci Sports 2016; 26: $550-556$

46 Chatterji U, Ashworth MJ, Lewis PL et al. Effect of total hip arthroplasty on recreational and sporting activity. ANZ journal of surgery 2004; 74 : $446-449$

47 Delasotta LA, Rangavajjula AV, Porat MD et al. What are young patients doing after hip reconstruction? The Journal of arthroplasty 2012; 27: $1518-1525 . e 1512$

48 Del Piccolo N, Carubbi C, Mazzotta A et al. Return to sports activity with short stems or standard stems in total hip arthroplasty in patients less than 50 years old. Hip Int 2016; 26 (Suppl 1): 48-51

49 Sandiford N, Muirhead-Allwood SK, Skinner JA. Return to sporting activity after Birmingham hip resurfacing arthroplasty: Mid term results. Indian journal of orthopaedics 2015; 49: 595-601

50 Banerjee $M$, Bouillon B, Banerjee $C$ et al. Sports activity after total hip resurfacing. Am J Sports Med 2010; 38: 1229-1236

51 Abe H, Sakai T, Nishii T et al. Jogging after total hip arthroplasty. Am J Sports Med 2014; 42: 131-137

52 Papaliodis DN, Photopoulos CD, Mehran N et al. Return to Golfing Activity After Joint Arthroplasty. Am J Sports Med 2016; DOI: 10.1177| 0363546516641917

53 D'Amico J, Betlach M, Senkarik R et al. Return to Golf Following Left Total Hip Arthroplasty in a Golfer Who is Right Handed. North American journal of sports physical therapy: NAJSPT 2007; 2: 251-261

54 Arbuthnot JE, McNicholas MJ, Dashti H et al. Total hip arthroplasty and the golfer: a study of participation and performance before and after surgery for osteoarthritis. The Journal of arthroplasty 2007; 22: 549 552
55 Seyler TM, Mont MA, Ragland PS et al. Sports activity after total hip and knee arthroplasty: specific recommendations concerning tennis Sports medicine (Auckland, NZ) 2006; 36: 571 - 583

56 Ho JC, Stitzlein RN, Green CJ et al. Return to Sports Activity following UKA and TKA. The journal of knee surgery 2016; 29: 254-259

57 Vielgut I, Leitner L, Kastner $N$ et al. Sports Activity after Low-contactstress Total Knee Arthroplasty - A long term follow-up study. Sci Rep 2016; 6: 24630

58 Mayr HO, Reinhold M, Bernstein A et al. Sports activity following total knee arthroplasty in patients older than 60 years. The Journal of arthroplasty 2015; 30: 46-49

59 Mont MA, Marker DR, Seyler TM et al. High-impact sports after total knee arthroplasty. The Journal of arthroplasty 2008; 23: 80-84

60 Bradbury N, Borton D, Spoo G et al. Participation in sports after total knee replacement. Am J Sports Med 1998; 26: 530 - 535

61 Chang MJ, Kang YG, Chung BJ et al. Why Patients Do Not Participate in Sports Activities After Total Knee Arthroplasty. Orthopaedic journal of sports medicine 2015; 3: 2325967115579171

62 Jackson JD, Smith J, Shah JP et al. Golf after total knee arthroplasty: do patients return to walking the course? Am J Sports Med 2009; 37 : $2201-2204$

63 Mont MA, Rajadhyaksha AD, Marxen JL et al. Tennis after total knee arthroplasty. Am J Sports Med 2002; 30: 163-166

64 Garcia GH, Liu JN, Mahony GT et al. Hemiarthroplasty Versus Total Shoulder Arthroplasty for Shoulder Osteoarthritis: A Matched Comparison of Return to Sports. Am J Sports Med 2016; DOI: 10.1177| 0363546516632527

65 Liu JN, Garcia GH, Mahony G et al. Sports after shoulder arthroplasty: a comparative analysis of hemiarthroplasty and reverse total shoulder replacement. Journal of shoulder and elbow surgery/American Shoulder and Elbow Surgeons [et al.] 2016; 25: 920-926

66 Schumann K, Flury MP, Schwyzer HK et al. Sports activity after anatomical total shoulder arthroplasty. Am J Sports Med 2010; 38: 2097-2105

67 Wang A, Doyle T, Cunningham G et al. Isokinetic shoulder strength correlates with level of sports participation and functional activity after reverse total shoulder arthroplasty. Journal of shoulder and elbow surgery/American Shoulder and Elbow Surgeons [et al.] 2016; 25: 1464 1469

68 Fink Barnes LA, Grantham WJ, Meadows MC et al. Sports activity after reverse total shoulder arthroplasty with minimum 2-year follow-up. American journal of orthopedics. Belle Mead, NJ 2015; 44: 68-72

69 Garcia GH, Taylor SA, DePalma BJ et al. Patient Activity Levels After Reverse Total Shoulder Arthroplasty: What Are Patients Doing? Am J Sports Med 2015; 43: 2816-2821

70 Simovitch RW, Gerard BK, Brees JA et al. Outcomes of reverse total shoulder arthroplasty in a senior athletic population. Journal of shoulder and elbow surgery/American Shoulder and Elbow Surgeons [et al.] 2015; $24: 1481$ - 1485

71 Bulhoff M, Sattler P, Bruckner T et al. Do patients return to sports and work after total shoulder replacement surgery? Am J Sports Med 2015; 43: 423-427

72 McCarty EC, Marx RG, Maerz D et al. Sports participation after shoulder replacement surgery. Am J Sports Med 2008; 36: 1577 - 1581

73 Papaliodis D, Richardson N, Tartaglione J et al. Impact of Total Shoulder Arthroplasty on Golfing Activity. Clinical journal of sport medicine: of ficial journal of the Canadian Academy of Sport Medicine 2015; 25: $338-340$

74 Laursen MK, Andersen JB, Andersen MM et al. Danish surgeons allow the most athletic activities after total hip and knee replacement. Eur J Orthop Surg Traumatol 2014; 24: 1571 -1577

75 Klein GR, Levine BR, Hozack WJ et al. Return to athletic activity after total hip arthroplasty. Consensus guidelines based on a survey of the Hip Society and American Association of Hip and Knee Surgeons. The Journal of arthroplasty 2007; 22: 171 - 175

76 Magnussen RA, Mallon WJ, Willems WJ et al. Long-term activity restrictions after shoulder arthroplasty: an international survey of experienced shoulder surgeons. Journal of shoulder and elbow surgery/ American Shoulder and Elbow Surgeons [et al.] 2011; 20: 281 - 289

77 Golant A, Christoforou D, Zuckerman JD et al. Return to sports after shoulder arthroplasty: a survey of surgeons' preferences. Journal of shoulder and elbow surgery/American Shoulder and Elbow Surgeons [et al.] 2012; 21: $554-560$

78 Lefevre N, Rousseau D, Bohu Y et al. Return to judo after joint replacement. Knee surgery, sports traumatology, arthroscopy: official journal of the ESSKA 2013; 21: 2889-2894 